\title{
The Heavy Atom Microwave Structure of
}

\section{2-Methyltetrahydrofuran}

\author{
Vinh Van ${ }^{a}$, Wolfgang Stahl ${ }^{a}$, and Ha Vinh Lam Nguyen ${ }^{* b}$ \\ ${ }^{\text {a } I n s t i t u t e ~ o f ~ P h y s i c a l ~ C h e m i s t r y, ~ R W T H ~ A a c h e n ~ U n i v e r s i t y, ~ L a n d o l t w e g ~ 2, ~ D-52074 ~ A a c h e n, ~}$
} Germany

\begin{abstract}
${ }^{\mathrm{b}}$ Laboratoire Interuniversitaire des Systèmes Atmosphériques (LISA), CNRS UMR 7583, Université Paris Est-Créteil, Université Paris Diderot, 61 avenue du Général de Gaulle, F-94010 Créteil cedex, France.
\end{abstract}

\section{* Corresponding Author}

Phone: +33 1451765 48; Email: lam.nguyen@lisa.u-pec.fr

\section{ABSTRACT}

The rotational spectra of 2-methyltetrahydrofuran have been observed using a pulsed molecular beam Fourier transform microwave spectrometer operating in the frequency range $2-26.5 \mathrm{GHz}$. Conformational analysis using quantum chemical calculations yields two stable conformers; both of them possess an envelope structure. The conformational transformation can occur via two different transition states. The Cremer-Pople notation for five-membered rings is chosen for describing the conformations. Only one conformer with equatorial position of the methyl group is assigned in the experimental spectrum. The fits of its parent species, ${ }^{13} \mathrm{C}$ - and ${ }^{18} \mathrm{O}$-isotopologues result in highly 
accurate molecular parameters, and enable the determination of a heavy atom $r_{s}$ structure using Kraitchman's equations. This experimentally determined structure is in excellent agreement with the structure calculated by anharmonic frequency calculations.

KEYWORDS: rotational spectroscopy, conformational analysis, isotopic substitution, structure determination, Kraitchman's equations

\section{INTRODUCTION}

Rotational spectroscopy in the gas phase has increasingly been applied to determine the structure of medium-sized molecules, which often exhibit complex conformational landscapes. ${ }^{1-3}$ Since the traditional method by isotopic substitutions is not always possible, the support of quantum chemistry becomes a helpful tool, whereby ab initio structures can be taken as references for a comparison of the experimental and calculated molecular parameters. On the other hand, quantum chemical calculations at different levels of theory often differ in a wide range, whereas atomic positions determined by rotational spectroscopy are highly precise, ${ }^{4}$ in particular when using the molecular beam Fourier transform microwave (MB-FTMW) technique. With high sensitivity and excellent experimental accuracy, it is possible to observe singly substituted isotopologues such as ${ }^{13} \mathrm{C},{ }^{34} \mathrm{~S}$, ${ }^{15} \mathrm{~N}$, and ${ }^{18} \mathrm{O}$ in natural abundances. Comparisons of experimentally and computationally derived molecular parameters can therefore validate the quality of various theoretical methods in characterizing the studied system.

In this paper, we will report on the heavy atom $r_{s}$ structure of 2-methyltetrahydrofuran (MTHF) studied by MB-FTMW spectroscopy, and compare it with results from quantum chemistry. MTHF is a promising environmentally friendly solvent and biofuel component, which is derived from renewable resources. Following the principles of Green Chemistry, MTHF has been evaluated as an eco-friendly substance in various fields like organometallics, ${ }^{5}$ metathesis, ${ }^{6}$ and biosynthesis. ${ }^{7}$ 
The methyl group in MTHF exhibits internal rotation with a rather high barrier of $13.12 \mathrm{~kJ} \cdot \mathrm{mol}^{-1}$ (i.e. $1097 \mathrm{~cm}^{-1}$ ) calculated at the $\operatorname{CCSD}(\mathrm{T}) / 6-311++\mathrm{G}(\mathrm{d}, \mathrm{p})$ level of theory. In the experimental spectrum, narrow torsional splittings only occur in a few transitions with high $J$ values.

\section{QUANTUM CHEMICAL CALCULATIONS}

\subsection{Geometry optimizations}

Several starting geometries of MTHF were created based on the structures of two conformers of cyclopentane used as a prototype. $^{8}$ Geometry optimizations were performed at the MP2/6$311++\mathrm{G}(\mathrm{d}, \mathrm{p})$ level of theory using the GAUSSIAN package. ${ }^{9}$ This level was chosen, since it yielded rotational constants which were close to the experimental values for other heterocyclic molecules. $^{10,11}$ We only considered the $(S)$-configuration, because enantiomers cannot be distinguished using our technique. The geometry optimizations signified two envelope conformers, called the equatorial and the axial conformer. Harmonic frequency calculations at the same level of theory were also carried out to decide, whether the optimized geometries are true minima or saddle points. Anharmonic frequency calculations resulted in the quartic centrifugal distortion constants (shown in Table 1) and the rotational constants $B_{0}$ of the vibrational ground state.

The envelope conformations can be described by the ring plane determined by the $\mathrm{O}_{2} \mathrm{C}_{3}, \mathrm{C}_{4}, \mathrm{C}_{5}$ atoms, and the envelope fold determined either by $\mathrm{C}_{2}, \mathrm{O}, \mathrm{C}_{3}, \mathrm{C}_{4}$ or $\mathrm{C}_{2}, \mathrm{C}_{3}, \mathrm{O}, \mathrm{C}_{5}$, as depicted in Figure 2. The dihedral angle $\varphi=\angle\left(\mathrm{O}, \mathrm{C}_{5}, \mathrm{C}_{4}, \mathrm{C}_{3}\right)$ of the ring plane is zero and both angles $\gamma=\angle$ $\left(\mathrm{C}_{2}, \mathrm{O}, \mathrm{C}_{3}, \mathrm{C}_{4}\right)$ and $\gamma^{\prime}=\angle\left(\mathrm{C}_{2}, \mathrm{C}_{3}, \mathrm{O}, \mathrm{C}_{5}\right)$ of the envelope fold are equal, if the ring structure is not distorted.

Similar to the case of methylcyclopentane, the equatorial orientation of the methyl group is lower in energy than the axial orientation due to steric effects, where the hydrogen atoms in the methyl group are closer to the hydrogen atoms of the ring. The equatorial conformer is illustrated in Figure 1; the Cartesian coordinates of both conformers are also available in Table S-1 in the Electronic Supporting 
Information (ESI). The equatorial conformer was afterwards re-optimized at different levels of theory using the HF, DFT, MP2, and CCSD methods and various basis sets. The calculated rotational constants are listed in Table S-2 in the ESI. If not otherwise stated, all values given from now on will refer to the MP2/6-311++G(d,p) level of theory.

\subsection{Transition states}

Using the Berny algorithm, ${ }^{12}$ two transition states (called ${ }^{2} \mathrm{~T}_{3}$ and $\mathrm{E}_{1}$ ) were found. We notice that in both ${ }^{2} \mathrm{~T}_{3}$ and $\mathrm{E}_{1}$, the ring structure is slightly distorted. The transition state energies were obtained from single-point calculations at the $\operatorname{CCSD}(\mathrm{T}) / 6-311++\mathrm{G}(\mathrm{d}, \mathrm{p})$ level of theory using the geometries optimized at the MP2/6-311++G(d,p) level. The envelope transition state $\mathrm{E}_{1}$ is $2.00 \mathrm{~kJ} \cdot \mathrm{mol}^{-1}$ higher in energy than the twist configuration ${ }^{2} \mathrm{~T}_{3}$. Their Cartesian coordinates are also available in Table S1. Figure 3 indicates the intramolecular conversion between the stable conformers via ${ }^{2} T_{3}$ and $E_{1}$. Since ${ }^{2} \mathrm{~T}_{3}$ is only $0.10 \mathrm{~kJ} \cdot \mathrm{mol}^{-1}$ higher in energy than the axial conformer, the barrier to convert the axial conformer in the equatorial conformer via ${ }^{2} \mathrm{~T}_{3}$ is very small.

\subsection{Cremer-Pople puckering parameters}

The Cremer-Pople puckering parameters ${ }^{13}$ for both conformers and the transition states are calculated using the program $\mathrm{RING}^{14}$ to characterize the ring geometries in more detail. The conformational space for a five-membered ring has two dimensions characterized by the puckering amplitude $q_{2}$ and the angle phase $\phi_{2} \cdot{ }^{13}$ The orientation of the methyl group as a ring substituent is described by the angle $\alpha$ between the mean plane of the puckered ring and the bond of the methyl group. ${ }^{15}$ Figure 4 illustrates possible conformations based on the model of Altona and Sundaralingam. ${ }^{16}$ The calculated as well as the experimental geometry of the equatorial conformer has an envelope structure $\mathrm{E}_{2}$ with significant distribution of the twist structure ${ }^{1} \mathrm{~T}_{2}$, and the methyl substituent in equatorial orientation with $q_{2}=0.39, \phi_{2}=32.4^{\circ}$, and $\alpha=67.7^{\circ}$ as well as $\mathrm{q}_{2}=0.40, \phi_{2}$ $=27.48^{\circ}$, and $\alpha=68.5^{\circ}$, respectively. The axial conformer is a $1: 1$ mixture of the envelope structure 
${ }^{2} \mathrm{E}$ and the twist structure ${ }^{2} \mathrm{~T}_{3}$ and has the methyl substituent in axial orientation with $\mathrm{q}_{2}=0.38, \phi_{2}=$ $226.6^{\circ}$, and $\alpha=10.1^{\circ}$. The axial orientation is higher in energy than the equatorial orientation due to steric effects. The lower energy transition state ${ }^{2} \mathrm{~T}_{3}$ has a more pronounced twist structure with $\mathrm{q}_{2}=$ $0.38, \phi_{2}=242.4^{\circ}$, and $\alpha=13.1^{\circ}$, whereas the transition state $\mathrm{E}_{1}$ has an envelope structure with $\mathrm{q}_{2}=$ $0.36, \phi_{2}=179.5^{\circ}$, and $\alpha=17.1^{\circ}$. In both of them, the methyl group is in axial orientation.

\section{MICROWAVE SPECTROSCOPY}

\subsection{Measurements}

MTHF was purchased from Alfa Aesar GmbH \& Co. KG, Karlsruhe, Germany with a stated purity of $99 \%$ and stabilized with approximately 150-400 ppm BHT (butylated hydroxytoluene). Additional purification was not necessary, since the vapor pressure of BHT is too low for an observation in the gas phase under the same conditions. The rotational spectra were measured with a MB-FTMW spectrometer operating in the frequency range $2-26.5 \mathrm{GHz}$. A detailed description is given in Refs. 17 and 18. A gas mixture of $1 \%$ substance in helium at a total pressure of 60 to 110 $\mathrm{kPa}$ was expanded through the nozzle into the cavity.

A broadband scan from 8.8 to $12.4 \mathrm{GHz}$ was recorded, which consists of automatically taken overlapping spectra with 50 co-added decays per each at a step width of $0.25 \mathrm{MHz}$. The scan indicates positions of the rotational transitions, which were remeasured afterwards at higher resolution. The typical line widths are $10-40 \mathrm{kHz}$; the measurement accuracy of isolated lines is approximately $2 \mathrm{kHz}$. Figure 5 illustrates some sections of a typical broadband scan and a high resolution spectrum.

\subsection{Spectral assignment}

We do not expect to observe the axial conformer in the spectrum because (i) there is an energy difference of $4.59 \mathrm{~kJ} \cdot \mathrm{mol}^{-1}$ between the two stable conformers of MTHF (calculated at the $\operatorname{CCSD}(\mathrm{T}) / 6-311++\mathrm{G}(\mathrm{d}, \mathrm{p})$ level of theory). The axial conformer is thus too high in energy to be 
observed under molecular beam conditions, where the rotational temperature is very low (about $2 \mathrm{~K}$ ) and (ii) the intramolecular conversion from the axial conformer to the equatorial conformer has a very low barrier of only $0.10 \mathrm{~kJ} \cdot \mathrm{mol}^{-1}$ (see section 2.2 ).

After assigning the equatorial conformer, all attempts to assign the axial conformer failed. Therefore, we believe that (i) in the supersonic expansion only the equatorial conformer exists due to collisional conformational relaxation and (ii) the remaining lines with much weaker intensity belong to vibrationally excited states occurring from ring puckering effects. Only the assignment procedure of the equatorial conformer is thus given here.

\subsubsection{Parent species}

The calculated dipole moment components were $\left|\mu_{a}\right|=0.69 \mathrm{D},\left|\mu_{b}\right|=1.60 \mathrm{D}$, and $\left|\mu_{c}\right|=0.62 \mathrm{D}$. Correspondingly, we expected strong $b$-type and medium intense $a$ - and $c$-type transitions. Nevertheless, we still began with searching for $a$-type transitions due to their characteristic pattern, which could be often assigned readily in the spectrum. At a rotational temperature of about $2 \mathrm{~K}$ of the molecular beam, transitions with low $J$ and $K$ values are often of highest intensity. Therefore, we started our assignment with the $a$-type $R$-branch transitions $2_{12} \leftarrow 1_{11}, 2_{02} \leftarrow 1_{01}$, and $2_{11} \leftarrow 1_{10}$. They were indeed three of the most intense lines in the broadband scan as can be recognized in Figure 6. The assignment of these lines fixed the $B$ and $C$ rotational constants. To determine the $A$ rotational constant, the $b$-type $Q$-branch transitions $2_{20} \leftarrow 2_{11}, 3_{21} \leftarrow 3_{12}, 4_{22} \leftarrow 4_{13}$, and $5_{23} \leftarrow 5_{14}$ were taken into account (also given in Figure 6). Afterwards, further lines up to $J=15$ (including $c$ type transitions) could be assigned and fitted. The molecular parameters were fitted using the XIAM program $^{19}$ and summarized in Table 1; the frequency list is available in Table S-3 in the ESI. 


\subsection{2. ${ }^{13}$ C-isotopologues}

After the most intense transitions were assigned to the equatorial conformer, a large number of lines with low to medium intensities remained in the scan. We assumed that these transitions belong to the ${ }^{13} \mathrm{C}$-isotopologues of MTHF in their natural abundances of $1 \%$.

By changing the mass of $\mathrm{a}^{12} \mathrm{C}$ atom to that of the ${ }^{13} \mathrm{C}$ atom, the rotational constants of the respective ${ }^{13} \mathrm{C}$ isotopologue could be obtained. We used the same transitions as mentioned in section 3.2.1 for the assignment process. Since the line density was quite high, we wrote a script which automatically generates XIAM inputs and fits to simplify the assignment. The calculated frequencies were close to those of the respective transitions of the main isotopologue, as can be recognized in Figure 7 . The calculated and experimental $a$-type $R$-branch frequencies differed by less than $10 \mathrm{MHz}$, whereas the $b$-type Q-branch is shifted by approximately $160 \mathrm{MHz}$ in the spectra of all isotopologues.

For all ${ }^{13} \mathrm{C}$-isotopologue, the rotational constants and four quartic centrifugal distortion constants could be determined with high accuracy, as summarized in Table 2; the frequency lists are also given in Table S-3.

\subsection{3. ${ }^{18}$ O-isotopologue}

The natural abundance of the ${ }^{18} \mathrm{O}$-isotopologue is $0.2 \%$. Its rotational transitions, therefore, did not appear in the scan like those of the ${ }^{13} \mathrm{C}$-isotopologues. Figure 5 visualizes the relative intensity of the parent species, ${ }^{13} \mathrm{C}$-, and ${ }^{18} \mathrm{O}$-isotopologues in the broadband scan. Whereas the $b$-type transitions mentioned in section 3.2.1 were discarded here, the same $a$-type $R$-branch transitions $2_{12} \leftarrow 1_{11}, 2_{02}$ $\leftarrow 1_{01}$, and $2_{11} \leftarrow 1_{10}$ as well as three other $a$-type lines $3_{13} \leftarrow 2_{12}, 3_{03} \leftarrow 2_{02}$, and $3_{12} \leftarrow 2_{11}$ were searched, since $a$-type transition frequencies could, in general, be much better predicted.

Scans of $\pm 5 \mathrm{MHz}$ around the predicted frequencies with overlapping spectra of 750 co-added decays per each and a step width of $0.2 \mathrm{MHz}$ were performed. In total, 14 transitions could be found and fitted. The quartic centrifugal distortion constants $\Delta_{J}$ and $\Delta_{K}$ were well determined, whereas the $\delta_{J}$ 
and $\delta_{K}$ were fixed. The frequency list for the ${ }^{18} \mathrm{O}$-isotopologue is also given in Table S-3, the fitted molecular parameters in Table 2.

\subsection{Internal rotation}

The methyl group in MTHF undergoes internal rotation and causes A-E splittings of all rotational lines. ${ }^{20}$ The barrier to internal rotation is calculated using various method-basis set combinations, as given in Table $\mathrm{S}-2$. The $\operatorname{CCSD}(\mathrm{T}) / 6-311++\mathrm{G}(\mathrm{d}, \mathrm{p})$ level of theory yields a barrier of $13.12 \mathrm{~kJ} \cdot \mathrm{mol}^{-1}$ $\left(1097 \mathrm{~cm}^{-1}\right)$, which is rather high. The splittings, if resolvable, are expected to be very small, in the order of a few tens of $\mathrm{kHz}$. In the experimental spectrum, only a few transitions at high $J$ values show A-E splittings. A typical spectrum with observable splitting is given in Figure S-F1 in the SI. We tried to fit the torsional splittings of 23 rotational transitions. Nevertheless, due to the small splittings, we did not obtain a reasonable fit. Furthermore, very small splittings often appear larger after Fourier transformation. The barrier to internal rotation was not well determined, and the standard deviation was four times larger than the measurement accuracy. Therefore, we completely neglected the effect of internal rotation in MTHF.

It should be noted that the A-E splittings are larger for excited torsional states, and the barrier to internal rotation could be much better determined if transitions from the excited torsional states were included. Unfortunately, these states are not observable in a supersonic jet.

\section{DISCUSSION}

\subsection{Results of the fits}

For the equatorial conformer, the rigid rotor fit including 111 rotational transitions has a standard deviation of $2.2 \mathrm{kHz}$, which is within the measurement accuracy. However, four sextic centrifugal distortion constants are needed to achieve this fit quality, while only transitions with $J \leq 15$ are included. Omitting these constants increases the standard deviation to $3.6 \mathrm{kHz}$, as shown in Fit II in Table S-4 in the ESI. By taking into account only transitions with $J \leq 6$, the standard deviation 
decreases to $1.4 \mathrm{kHz}$ (Fit III, also in Table S-4). As mentioned in section 3.3, the internal rotation of the methyl group causes splittings of about $20 \mathrm{kHz}$ for some high $J$ transitions. In such cases, we neglect the E component and only include the A component in the fit. This approximate treatment partly explains the increase of the standard deviation of the fit, when transitions with $J>6$ are considered. It might also be the reason that some sextic centrifugal distortion constants improve the fit. The quartic centrifugal distortion constants are in a normal order of magnitude for molecules with comparable rotational constants, and there is no indication for an unusual floppiness of the molecule. The constants obtained from anharmonic frequency calculations are close to the experimental values for $\Delta_{J}, \delta_{J}$, and $\delta_{K}$. The deviations for $\Delta_{J K}, \Delta_{K}$, and all sextic centrifugal distortion constants are remarkable, probably due to the effects of small vibration rotation interactions which have been taken into account in the calculations for these constants.

The experimentally deduced rotational constants are compared with those from quantum chemical calculations and listed in Table S-2 in the ESI. Because the experimental constants $B_{0}$ refer to the ground vibrational state ( $r_{0}$ structure), whereas the theoretical constants $B_{\mathrm{e}}$ refer to the equilibrium geometry ( $r_{\mathrm{e}}$ structure), the values usually agree only within $1 \%$. We find the best agreement at the B3LYP/cc-pVQZ level of theory. The MP2 method in combination with triple zeta basis sets provides excellent results for the $B$ and $C$ rotational constants but a larger deviation for the $A$ rotational constant. The M06-2X functional is specifically designed for taking into account longrange dispersive interactions and yields also good results in this case. We note that the rotational constants of the ground vibrational state can be predicted by anharmonic frequency calculations, as given in Table 1. At the MP2/6-311++G(d,p) level of theory, the calculated $A$ rotational constant agrees well with the experimental values; on the other hand, the $B$ and $C$ constants are much worse comparing to the results from geometry optimizations (see Table S-2). Anharmonic frequency calculations at other levels of theory cannot be done because of the limitation of our calculations. 


\subsection{Microwave structure determination}

The structure determination can be performed using the experimentally deduced rotational constants of the parent species and the ${ }^{13} \mathrm{C}$ and ${ }^{18} \mathrm{O}$-isotopologues. The isotopic shifts allow us to calculate the heavy atom (carbon and oxygen) positions with respect to the center of mass of the parent species using Kraitchman's equations ${ }^{21}$ as implemented in the program KRA and EVAL, which are available from the PROSPE database. ${ }^{22}$ The uncertainties are calculated using Costain's rule, saying that the uncertainty is inversely proportional to the center of mass distance. ${ }^{23}$ The signs of the atom coordinates have been chosen from the coordinates calculated at the MP2/6-311++G(d,p) level. This determined $r_{s}$ structure is in excellent agreement with the vibrational averaged structure $\left(r_{\mathrm{z}}\right)$ calculated by anharmonic frequency calculations, as shown in Figure 8. The Cartesian coordinates of the $r_{\mathrm{z}}$ geometry are given in Table S-1 in the ESI.

The experimental bond lengths, angles, and dihedral angles are indicated in Table 3. By regarding both, Figure 7 and Figure 8, we note that the frequency shifts from the parent transitions increase with the distance of the substituted carbon atom to the center of mass.

The microwave structure yields the experimental ring plane dihedral angle $\varphi$ as well as the envelope fold angles, which characterize the envelope structure of MTHF as mentioned in section 2.1. The ring plane is indeed distorted by $\varphi=-6.48(21)^{\circ}$, resulting in a "near-envelope" conformation of the observed conformer. The envelope fold angles are $\gamma=135.715(48)^{\circ}$ and $\gamma^{\prime}=139.950(54)^{\circ}$. These values reasonably agree with the calculated angles of $\varphi=-3.66^{\circ}, \gamma=137.46^{\circ}$, and $\gamma^{\prime}=139.90^{\circ}$. The experimental Cremer-Pople parameters $\mathrm{q}_{2}=0.40, \phi_{2}=27.5^{\circ}$, and $\alpha=68.5^{\circ}$ show that the equatorial conformer has a more twist character (see Figure 4) than the structure calculated at the MP2/6$311++\mathrm{G}(\mathrm{d}, \mathrm{p})$ level of theory $\left(\mathrm{q}_{2}=0.39, \phi_{2}=32.4^{\circ}\right.$ and $\left.\alpha=67.7^{\circ}\right)$. 


\section{CONCLUSION}

Geometry optimizations of MTHF using quantum chemical calculations yielded two stable conformers (equatorial and axial); both of them have an envelope structure. The intramolecular conversion from the axial conformer to the energetically more favorable equatorial conformer can occur via two transition states; one has an envelope and the other one a twist structure. In the microwave spectra recorded using the MB-FTMW technique, only the equatorial conformer could be observed. Rotational transitions of all heavy atom isotopologues were assigned, resulted in highly accurate rotational constants, centrifugal distortion constants, and the heavy atom positions with respect to the center of mass. The observed spectra could be reproduced within our measurement accuracy of $2 \mathrm{kHz}$.

\section{ACKNOWLEDGMENT}

The authors thank the Land Nordrhein-Westfalen for financial supports. V.V. thanks the Fonds der Chemischen Industrie (FCI) for a Ph.D. fellowship. Simulations were performed with computing resources granted by JARA-HPC from the RWTH Aachen University under the project jara0124.

\section{REFERENCES}

(1) Shipman, S. T.; Neill, J. L.; Suenram, R. D.; Muckle, M. T.; Pate, B. H. Structure Determination of Strawberry Aldehyde by Broadband Microwave Spectroscopy: Conformational Stabilization by Dispersive Interactions. J. Phys. Chem. Lett. 2011, 2, 443-448.

(2) Grabow, J.-U.; Mata, S.; Alonso, J. L.; Peña, I.; Blanco, S.; López, J. C.; Cabezas, C. Rapid Probe of the Nicotine Spectra by High-Resolution Rotational Spectroscopy. Phys. Chem. Chem. Phys. 2011, 13, 21063-21069.

(3) Attig, T.; Sutikdja, L. W.; Kannengießer, R.; Kleiner, I.; Stahl, W. The Microwave Spectrum of n-Butylacetate. J. Mol. Spectrosc. 2013, 284-285, 8-15. 
(4) Martinez O.; Crabtree, K. N.; Gottlieb, C. A.; Stanton, J. F.; McCarthy, M. C. An Accurate Molecular Structure of Phenyl, the Simplest Aryl Radical. Angew. Chem. Int. Ed. 2015, 54, $1808-1811$.

(5) Aycock, D. F. Solvent Applications of 2-Methyltetrahydrofuran in Organometallic and Biphasic Reactions. Org. Process Res. Dev. 2007, 11, 156-159.

(6) Smoleń, M.; Kędziorek, M.; Grela, K. 2-Methyltetrahydrofuran: Sustainable Solvent for Ruthenium-Catalyzed Olefin Metathesis. Catal. Commun. 2014, 44, 80-84.

(7) Duan, Z.-Q.; Hu, F. Efficient Synthesis of Phosphatidylserine in 2-Methyltetrahydrofuran. J. Biotechnol. 2013, 163, 45-49.

(8) Lightner, D. A.; Gurst, J. E. Organic Conformational Analysis and Stereochemistry from Circular Dichroism Spectroscop. J. Wiley-VCH, New York, 2000.

(9) Frisch, M. J.; Trucks, G. W.; Schlegel, H. B.; Scuseria, G. E.; Robb, M. A.; Cheeseman, J. R.; Scalmani, G.; Barone, V.; Mennucci, B.; Petersson, G. A.; et al. Gaussian 09, Revision A.02, Gaussian, Inc., Wallingford CT, 2009.

(10) Mouhib, H.; Stahl, W.; Lüthy, M.; Büchel, M.; Kraft, P. Cassis Odor through Microwave Eyes: Olfactory Properties and Gasphase Structures of all the Cassyrane Stereoisomers and its Dihydro Derivative. Angew. Chem. Int. Ed. 2011, 50, 5576-5580.

(11) Van, V.; Dindic, C.; Nguyen, H. V. L.; Stahl, W. Conformational Transformations of SulfurContaining Rings: 2-Methyltetrahydrothiophene Gas-Phase Structures. ChemPhysChem. 2015, 16, 291-294.

(12) Schlegel, H. B. Optimization of Equilibrium Geometries and Transition Structures. $J$. Comput. Chem. 1982, 3, 214-218.

(13) Cremer D.; Pople, J. A. A General Definition of Ring Puckering Coordinates. J. Am. Chem. Soc. 1975, 97, 1354-1358. 
(14) Cremer, D. RING - A Coordinate Transformation Program for Evaluating the Degree and Type of Puckering of a Ring Compound. Quantum Chemical Program Exchange 1975, 288, 1-8. Available at https://sites.smu.edu/dedman/catco/ring-puckering.html.

(15) Cremer, D. A General Definition of Ring Substituent Positions", Isr. J. Chem. 1980, 20, $12-19$.

(16) Altona, C.; Sundaralingam, M. Conformational analysis of the sugar ring in nucleosides and nucleotides. New description using the concept of pseudorotation. J. Am. Chem. Soc. 1972, 94, $8205-8212$.

(17) Andresen, U.; Dreizler, H.; Grabow, J.-U.; Stahl, W. An Automatic Molecular Beam Microwave Fourier Transform Spectrometer. Rev. Sci. Instrum. 1990, 61, 3694-3699.

(18) Grabow, J.-U.; Stahl, W.; Dreizler, H. A Multioctave Coaxially Oriented Beam Resonator Arrangement Fourier-Transform Microwave (COBRA-FTMW) Spectrometer. Rev. Sci. Instrum. 1996, 67, 4072-4084.

(19) Hartwig, H.; Dreizler, H. The Microwave Spectrum of trans-2,3-Dimethyloxirane in Torsional Excited States. Z. Naturforsch. 1996, 51a, 923-932.

(20) Dreizler, H. Gruppentheoretische Betrachtungen zu den Mikrowellenspektren von Molekülen mit zwei behindert drehbaren dreizählig-symmetrischen Molekülgruppen. Z. Naturforsch. 1961, $16 a, 1354-1367$.

(21) Kraitchman, J. Determination of Molecular Structure from Microwave Spectroscopic Data. Am. J. Phys. 1953, 21, 17-24.

(22) Kisiel, Z.; PROSPE-Programs for ROtational SPEctroscopy, available at http://info.ifpan.edu.pl/ kisiel/prospe.htm.

(23) Costain, C. C. Further comments on the accuracy of $r_{s}$ substitution structures. Trans. Am. Crystallogr. Assoc. 1966, 2, 157-164. 


\section{TABLES}

Table 1. Experimentally deduced molecular parameters of the equatorial conformer of MTHF compared to the $r_{0}$ vibrational ground state values calculated by anharmonic frequency calculation at the MP2/6-311++G(d,p) level of theory.

\begin{tabular}{llll}
\hline \hline Par. $^{\mathrm{a}}$ & $\mathrm{Unit}$ & Fit I $(J \leq 15)$ & $\mathrm{MP} 2$ \\
\hline$A$ & $\mathrm{GHz}$ & $6.59156823(22)$ & 6.5798 \\
$B$ & $\mathrm{GHz}$ & $3.170370175(84)$ & 3.1425 \\
$C$ & $\mathrm{GHz}$ & $2.367116054(88)$ & 2.3457 \\
$\Delta_{J}$ & $\mathrm{kHz}$ & $0.3312(13)$ & 0.257 \\
$\Delta_{J K}$ & $\mathrm{kHz}$ & $0.0641(35)$ & 0.761 \\
$\Delta_{K}$ & $\mathrm{kHz}$ & $2.855(20)$ & 0.849 \\
$\delta_{J}$ & $\mathrm{kHz}$ & $0.05881(44)$ & 0.057 \\
$\delta_{K}$ & $\mathrm{kHz}$ & $0.5322(69)$ & 0.561 \\
$H_{J K}$ & $\mathrm{~Hz}$ & $-0.788(96)$ & $-0.163 \cdot 10^{-3}$ \\
$H_{K J}$ & $\mathrm{~Hz}$ & $2.83(35)$ & $0.111 \cdot 10^{-3}$ \\
$h_{J K}$ & $\mathrm{~Hz}$ & $0.331(46)$ & $0.059 \cdot 10^{-3}$ \\
$h_{K}$ & $\mathrm{~Hz}$ & $-2.84(55)$ & $2.367 \cdot 10^{-3}$ \\
$\sigma^{b}$ & $\mathrm{kHz}$ & 2.2 & \\
$N^{c}$ & & 111 & \\
\hline
\end{tabular}

${ }^{\text {a }}$ All parameters refer to the principal axis system. Watson's A reduction in $\mathrm{I}^{\mathrm{r}}$ representation was used. ${ }^{\mathrm{b}}$ Standard deviation of the fit. ${ }^{\mathrm{c}}$ Number of lines.

Table 2. Experimentally deduced molecular parameters of different isotopologues of the equatorial conformer. The atoms are numbered according to Figure 1.

\begin{tabular}{llllllll}
\hline \hline Par. & Unit & ${ }^{18} \mathrm{O}(1)$ & ${ }^{13} \mathrm{C}(2)$ & ${ }^{13} \mathrm{C}(3)$ & ${ }^{13} \mathrm{C}(4)$ & ${ }^{13} \mathrm{C}(5)$ & ${ }^{13} \mathrm{C}(6)$ \\
\hline$A$ & $\mathrm{GHz}$ & $6.3962301(30)$ & $6.57970964(61)$ & $6.46187441(69)$ & $6.55107431(70)$ & $6.53423752(71)$ & $6.59028755(61)$ \\
$B$ & $\mathrm{GHz}$ & $3.16966061(63)$ & $3.15713442(33)$ & $3.17000430(43)$ & $3.12372913(38)$ & $3.13465551(41)$ & $3.08086383(37)$ \\
$C$ & $\mathrm{GHz}$ & $2.34229427(70)$ & $2.36130876(29)$ & $2.34994423(31)$ & $2.33611689(36)$ & $2.34012997(37)$ & $2.31701145(35)$ \\
$\Delta_{J}$ & $\mathrm{kHz}$ & $0.332(29)$ & $0.3318(69)$ & $0.3355(82)$ & $0.329(12)$ & $0.3235(76)$ & $0.3143(72)$ \\
$\Delta_{K}$ & $\mathrm{kHz}$ & $2.53(53)$ & $3.021(51)$ & $2.781(53)$ & $2.696(55)$ & $2.610(59)$ & $2.997(50)$ \\
$\delta_{J}$ & $\mathrm{kHz}$ & $0.06016^{\mathrm{b}}$ & $0.0665(30)$ & $0.0663(35)$ & $0.0553(27)$ & $0.0496(44)$ & $0.0513(48)$ \\
$\delta_{K}$ & $\mathrm{kHz}$ & $0.580^{\mathrm{b}}$ & $0.596(26)$ & $0.662(39)$ & $0.544(27)$ & $0.709(43)$ & $0.617(56)$ \\
$\sigma^{\mathrm{c}}$ & $\mathrm{kHz}$ & 3.0 & 2.3 & 2.3 & 2.8 & 2.4 & 3.0 \\
$\mathrm{~N}^{\mathrm{d}}$ & & 14 & 33 & 31 & 37 & 30 & 37 \\
\hline
\end{tabular}

${ }^{\mathrm{a}}$ All parameters refer to the principal axis system. Watson's A reduction in $\mathrm{I}^{\mathrm{r}}$ representation was used. ${ }^{b}$ Fixed to the value from Fit III in Table S-4 in the SI, since the two fits are most similar. ${ }^{c}$ Standard deviation of the fit. ${ }^{\mathrm{d}}$ Number of lines. 
Table 3a. Experimental heavy atom positions of the equatorial conformer obtained by isotopic substitutions and using Kraitchmann's equations implemented in the program KRA. ${ }^{21}$ The atoms are numbered according to Figure 1.

\begin{tabular}{llll}
\hline \hline & \multicolumn{1}{c}{$|\mathrm{a}| / \AA$} & \multicolumn{1}{c}{$|\mathrm{b}| / \AA$} & \multicolumn{1}{c}{$|\mathrm{c}| / \AA$} \\
\hline O1 & $0.104(15)$ & $1.0792(14)$ & $0.1747(86)$ \\
C2 & $0.7290(21)$ & $0.051(30)$ & $0.3774(40)$ \\
C3 & $0.140(11)$ & $1.2464(12)$ & $0.039(39)$ \\
C4 & $1.54132(97)$ & $0.6932(22)$ & $0.104(15)$ \\
C5 & $1.3428(12)$ & $0.8240(19)$ & $0.118(13)$ \\
C6 & $2.15737(70)$ & $0.018(83)$ & $0.123(13)$ \\
\hline
\end{tabular}

Table 3b. Experimental bond lengths, bond angles, and dihedral angles of the equatorial conformer obtained using the program EVAL. ${ }^{21}$

\begin{tabular}{lllllr}
\hline \hline \multicolumn{2}{c}{ Bond lengths / $\AA$} & \multicolumn{2}{c}{ Bond angles / $^{\circ}$} & \multicolumn{2}{c}{ Dihedral angles / } \\
\hline $\mathrm{O} 1-\mathrm{C} 2$ & $1.404(21)$ & $\mathrm{O} 1-\mathrm{C} 2-\mathrm{C} 3$ & $105.54(89)$ & $\mathrm{C} 5-\mathrm{C} 4-\mathrm{C} 3-\mathrm{C} 2$ & $-18.26(13)$ \\
$\mathrm{C} 2-\mathrm{C} 3$ & $1.536(21)$ & $\mathrm{C} 2-\mathrm{C} 3-\mathrm{C} 4$ & $102.34(94)$ & $\mathrm{C} 4-\mathrm{C} 3-\mathrm{C} 2-\mathrm{C} 6$ & $157.13(18)$ \\
$\mathrm{C} 3-\mathrm{C} 4$ & $1.5132(86)$ & $\mathrm{C} 3-\mathrm{C} 4-\mathrm{C} 5$ & $104.07(20)$ & $\mathrm{C} 4-\mathrm{C} 3-\mathrm{C} 2-\mathrm{O} 1$ & $39.483(67)$ \\
$\mathrm{C} 4-\mathrm{C} 5$ & $1.5304(23)$ & $\mathrm{C} 4-\mathrm{C} 5-\mathrm{O} 1$ & $107.01(23)$ & $\mathrm{C} 3-\mathrm{C} 4-\mathrm{C} 5-\mathrm{O} 1$ & $-6.48(21)$ \\
$\mathrm{O} 1-\mathrm{C} 5$ & $1.498(12)$ & $\mathrm{C} 5-\mathrm{O} 1-\mathrm{C} 2$ & $102.49(68)$ & $\mathrm{C} 4-\mathrm{C} 5-\mathrm{O} 1-\mathrm{C} 2$ & $31.081(70)$ \\
$\mathrm{C} 2-\mathrm{C} 6$ & $1.5139(41)$ & $\mathrm{O} 1-\mathrm{C} 2-\mathrm{C} 6$ & $105.8(30)$ & $\mathrm{C} 5-\mathrm{O} 1-\mathrm{C} 2-\mathrm{C} 3$ & $-43.334(58)$ \\
& & $\mathrm{C} 3-\mathrm{C} 2-\mathrm{C} 6$ & $117.4(32)$ & $\mathrm{C} 5-\mathrm{O} 1-\mathrm{C} 2-\mathrm{C} 6$ & $-168.49(31)$ \\
& & & & $\mathrm{C} 5-\mathrm{O} 1-\mathrm{C} 3-\mathrm{C} 2$ & $135.715(48)$ \\
& & & & $\mathrm{C} 4-\mathrm{C} 3-\mathrm{O} 1-\mathrm{C} 2$ & $-139.950(54)$ \\
\hline
\end{tabular}




\section{FIGURES}

Figure 1. The optimized geometry in the principal axes of inertia of the most stable conformer of MTHF calculated at the MP2/6-311++G(d,p) level of theory. Left-hand side: view along the $c$-axis; right-hand side: view along the $b$-axis. The circled dot and cross indicate that the axis direction points out of and into the paper plane, respectively. The blue arrow presents the dipole moment vector.

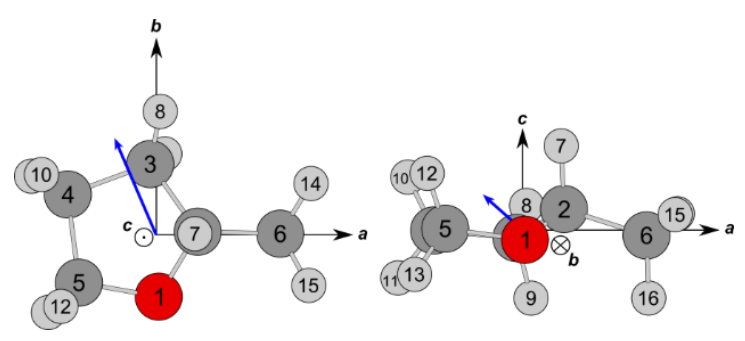

Figure 2. The envelope conformation is described by the dihedral angles angle $\varphi=\angle\left(\mathrm{O}, \mathrm{C}_{5}, \mathrm{C}_{4}, \mathrm{C}_{3}\right)$ of the ring plane and two angles $\gamma=\angle\left(\mathrm{C}_{2}, \mathrm{O}, \mathrm{C}_{3}, \mathrm{C}_{4}\right)$ and $\gamma^{\prime}=\angle\left(\mathrm{C}_{2}, \mathrm{C}_{3}, \mathrm{O}, \mathrm{C}_{5}\right)$, which describe the envelope flap. If the ring structure is not distorted, $\varphi=0$ and $\gamma=\gamma^{\prime}$.

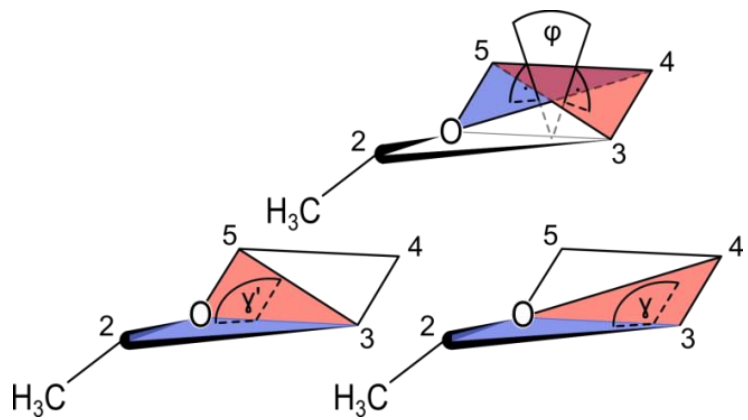


Figure 3. The intramolecular conversion between two stable envelope conformers (equatorial and axial) via one twist $\left({ }^{2} \mathrm{~T}_{3}\right)$ and one envelope $\left(\mathrm{E}_{1}\right)$ transition state. The energies at the CCSD(T)/6$311++\mathrm{G}(\mathrm{d}, \mathrm{p})$ are related to the lowest energetic the equatorial conformer with its absolute energy $\mathrm{E}$ $=-271.1307051$ Hartree. ${ }^{2} \mathrm{~T}_{3}$ is only $0.10 \mathrm{~kJ} \cdot \mathrm{mol}^{-1}$ higher in energy than the stable axial conformer. This facilitates the conversion from the axial conformer to the equatorial conformer.

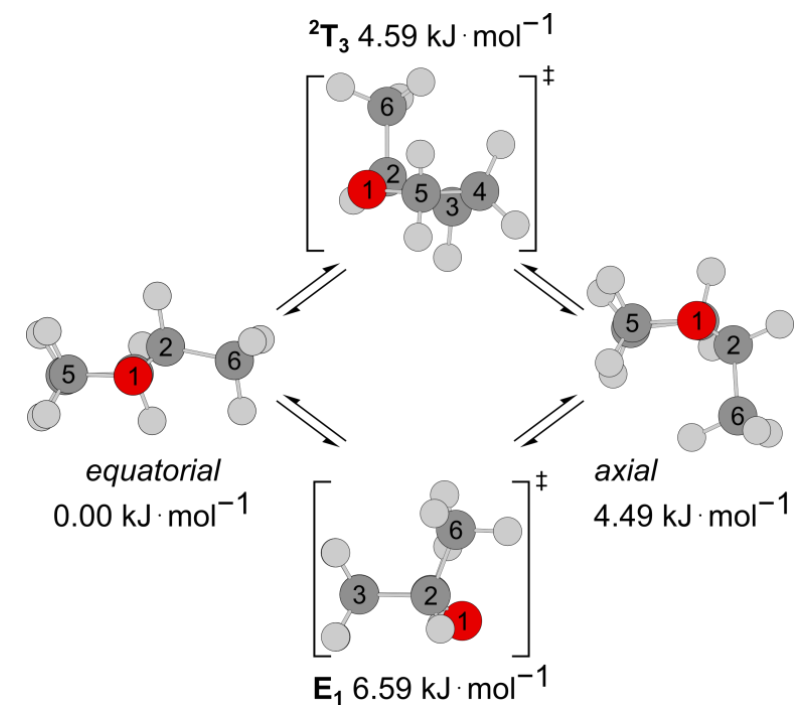


Figure 4. Conformational landscape of MTHF using two Cremer-Pople parameters $q_{2}$ (puckering amplitude) and $\phi_{2}$ (angle phase). The nomenclature is based on the Twist or Envelope geometry and the positions of the atoms which are above $(+)$ and below $(-)$ the ring plane. At the center, $q_{2}=0$ corresponds to a planar ring. Stable conformations are illustrated as dots and transition states as stars.

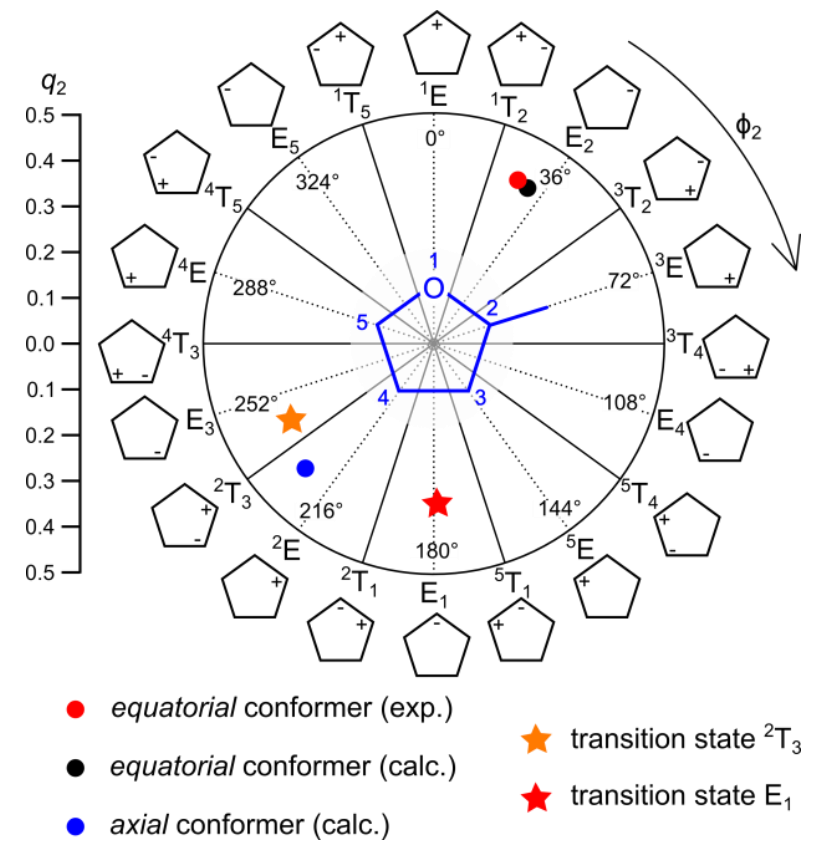


Figure 5. Upper diagram: Some sections of a typical broadband scan, where overlapping spectra are automatically taken at a step width of $0.25 \mathrm{MHz}$ with 50 co-added decays per each spectrum. The relative intensities on a logarithmic scale of the main, ${ }^{13} \mathrm{C}$-, and ${ }^{18} \mathrm{O}$-isotopologue are indicated. Lower diagram: A high resolution spectrum of the $2_{20} \leftarrow 2_{11}$ transition obtained after two co-added free induction decays (FIDs) with a line width of approximately $42 \mathrm{kHz}$. The splitting is due to the Doppler effect.

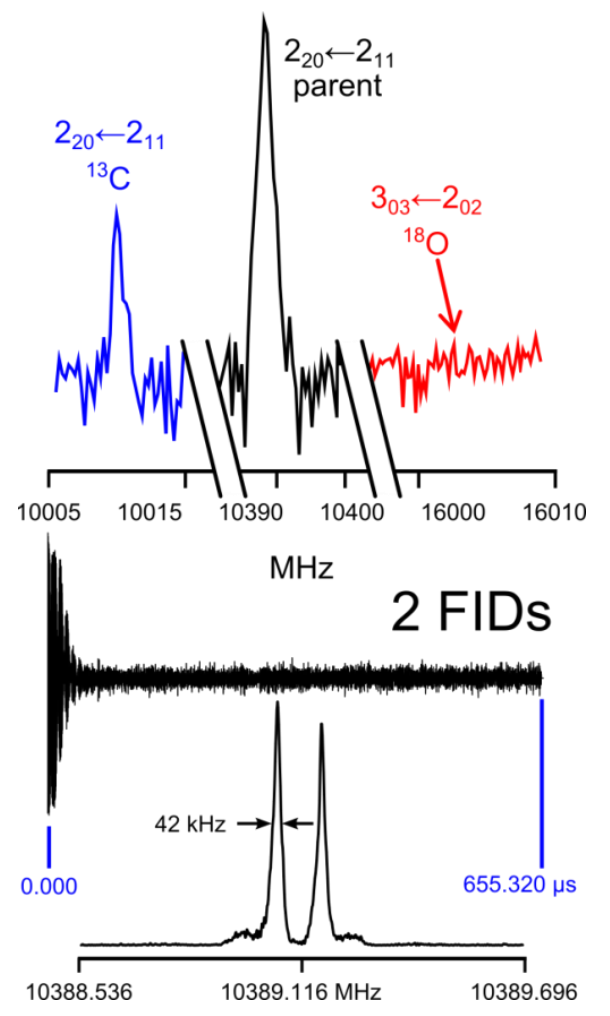


Figure 6. a) Upper trace: Experimental broadband scan in the frequency range from 9000 to 12000 MHz. Three $a$-type $R$-branch transitions $2_{12} \leftarrow 1_{11}, 2_{02} \leftarrow 1_{01}$, and $2_{11} \leftarrow 1_{10}$ of the parent species of the equatorial conformer are marked in red, some assigned $b$-type $Q$-branch transitions in blue. b) Lower trace: Predicted spectrum of the equatorial conformer using the rotational constants calculated at the MP2/6-311++G(d,p) level of theory. The calculated $a-, b$-, and $c$-type transitions are colored in red, blue, and orange, respectively.

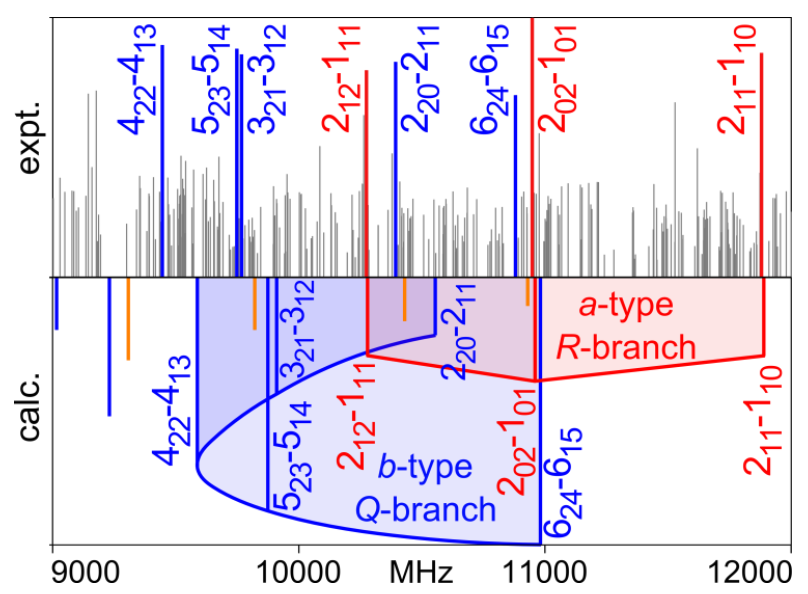


Figure 7. Predicted spectra of the ${ }^{13} \mathrm{C}$ and ${ }^{18} \mathrm{O}$-isotopologues compared to that of the parent species using the rotational constants calculated at the MP2/6-311++G(d,p) level. The substituted ${ }^{13} \mathrm{C}$ atom is numbered according to Figure 1.

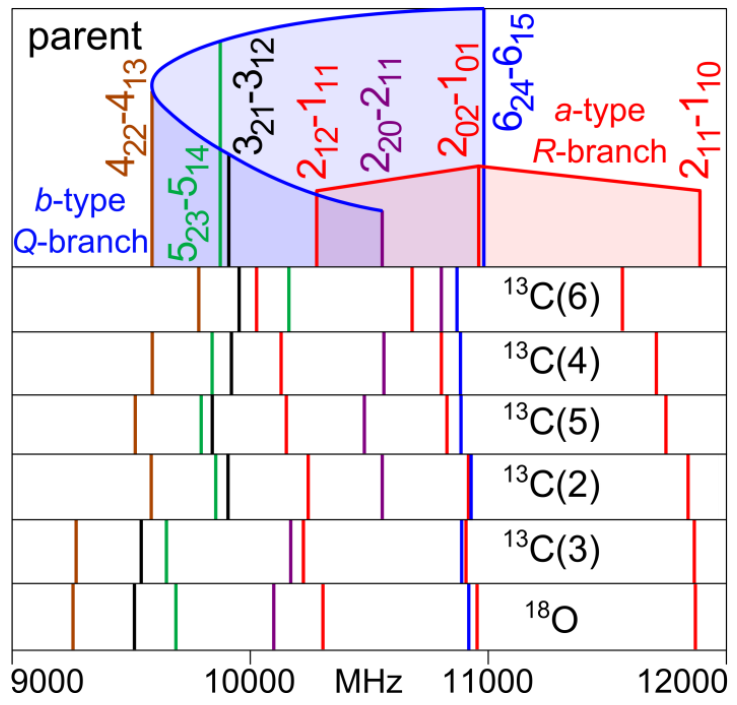

Figure 8. Comparison of the $r_{\mathrm{s}}$ microwave structure of MTHF (in blue) determined by heavy atom substitutions with atom positions (carbon in gray, oxygen in red) of the vibrational averaged geometry calculated by anharmonic frequency calculations at the MP2/6-311++G(d,p) level of theory. The indicated experimental bond lengths are given in $\AA$. Note that the further away the carbon atom is from the center of mass, the larger the frequency shift is from the parent transition (see Figure 7 for comparison).

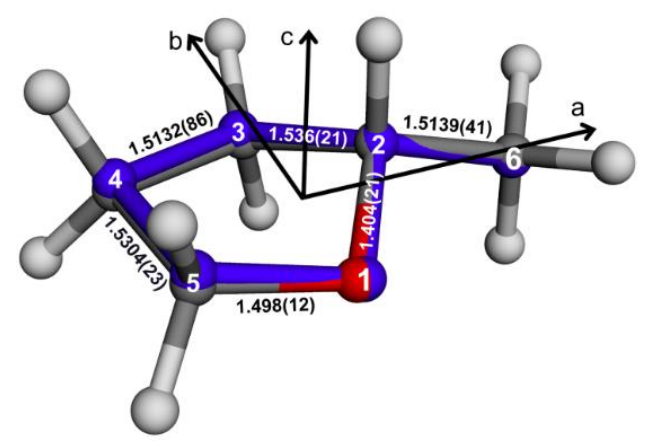


TABLE OF CONTENTS IMAGE

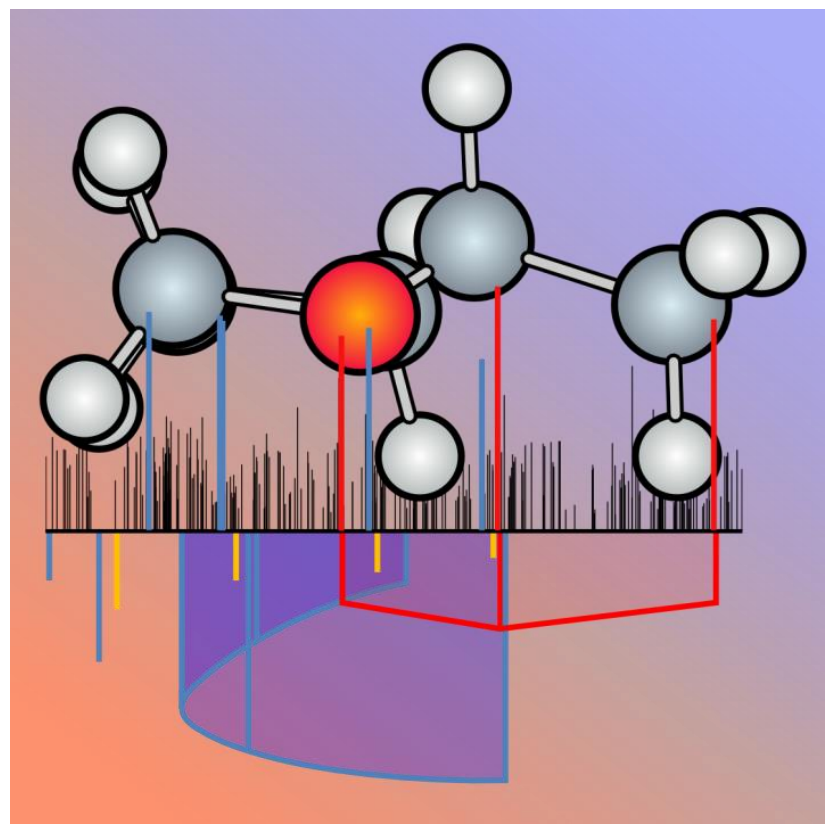

